\title{
Health disparity, comorbidity, and management of patients with diabetes by the physical therapists
}

\author{
Shetty A*, Alexander-Palacios B, Eckert C and Murphy M \\ Doctor of Physical Therapy Program, University of Saint Mary, Leavenworth, Kansas, USA
}

\begin{abstract}
Diabetes is the six most common cause of morbidity and mortality in the United States. African Americans in the United States are more likely to have diabetesrelated complications and number of amputations. The purpose of this study was to: 1) To assess the utilization of outpatient services by blacks with diabetes compared with whites 2) To investigate the course of treatment leading to amputation of blacks and whites with diabetes. Data was collected through the Medicare database obtained from the Center for Medicare and Medicaid Services (CMS). Inpatient and outpatient files from the Medicare database were used to collect data on the number of hospitalizations, the length of stay, the number of discharges to skilled nursing facilities (SNF), the number of doctors' visits and number of physical therapy visits for both groups. A t-test was used to compare both groups and a Chi-Square test was used to assess the frequency of occurrence of amputation in both groups. Significant differences in the clinical management of diabetes in whites and blacks were noted. Blacks had higher number of hospitalizations than whites, longer hospital stays, higher rate of discharge to SNFs and a higher rate of amputations than their white counterparts. Outpatient visits for Physical Therapy services for blacks was significantly lower than for whites. The results of the study show that there is a health disparity in the clinical management of diabetes for blacks as compared with whites in the United States in the absence of health insurance coverage difference. The disparity in outpatient physical therapy visits needs to be further investigated. The path to the elimination of health disparity is paramount in the delivery of quality of care to all and consistent with best practice.
\end{abstract}

\section{Introduction}

The prevalence of diabetes mellitus continues to rise worldwide in people of all ages and races. It estimated that diabetes affects more than 25 million Americans and ranks among the top ten most expensive medical conditions in the United States [1,2]. As a result of the progression and impact of the disease, the growing costs for national health care systems, and the chronic complications associated with the disease, diabetes mellitus has raised concern in the health care field. In addition, there has been great debate on what it means to receive optimal healthcare and specifically, who exactly has access to that optimal care. Also, minorities continue to statistically fall behind Caucasians when healthcare is concerned.

There are several risk factors that predispose individuals to diabetes. Risk factors include weight gain, obesity, sedentary lifestyle, high blood pressure, increasing age, ethnicity, unhealthy diet, and family history of the condition [2]. Diabetes can affect individuals of any race; however, research shows that minorities, specifically African Americans, are at an increased risk for developing the disease and its related comorbidities when compared to Caucasians [2]. Individuals with diabetes are likely to experience several complications as a result of the disease. These complications include hypertension, lower limb amputation, depression, kidney disease, and stroke. All of these previously mentioned complications can lead to an increase in hospital stays, doctor visits, physical therapy visits, individuals living in a skilled nursing facility, and risk of death. The higher incidence of complications in the African American population with diabetes could be related to the decrease in their amount of access and usage of the healthcare system [2]. A combination of these things contributes to lowering the life expectancy of individuals with diabetes mellitus by 5-12 years [3].
The study's overall goal is to understand the primary factors of diabetes mellitus and other healthcare concerns and to provide guidance for future community-level and macro-level programs to prevent and control diabetes mellitus and other related metabolic disorders. The specific objectives of this research were: (1) to identify differences between African Americans and Caucasians in the United States, from 2006-2008 and 2009-2011 with regard to complications associated with diabetes, (2) to assess if and how socioeconomic factors affect health-related behaviors, metabolic disorders, and hypertension, especially in African Americans as compared to Caucasians, (3) if differences exist between African Americans and Caucasians exist, to gather data that might elucidate, how and why these differences exist, and (4) to identify common characteristics or risk factors within the community of African Americans and Caucasians leading to diabetes. We hypothesized that differences related to the complications associated with diabetes will exist between African Americans and Caucasians as a result of socioeconomic factors related to their lifestyle, education, and access to healthcare.

\section{Methods}

The number of hospitalizations, length of stay, the number of outpatient and physical therapy visits for the two groups was compared using a T-test for independent samples at 0.05 level of significance. The frequency of occurrence of amputations for both groups was assessed using the Chi-Square test at the 0.05 level of significance. Data was

Correspondence to: Anand Shetty, Doctor of Physical Therapy Program, University of Saint Mary, Leavenworth, Kansas, USA, E-mail: anand.shetty@yahoo.com

Key words: type 2 diabetes, health care, African Americans, physical therapy

Received: July 24, 2016; Accepted: August 17, 2016; Published: August 22, 2016 
collected through the Medicare database obtained from the Center for Medicare and Medicaid Services (CMS). A random sample of patients with type 2 diabetes was collected from 2006-2008 and 2009-2011 using files derived from inpatient charts and insurance carrier data and analyzed by independent T-test. The sample included men and women; 181,857 Caucasians, with a mean age of $69.4 \pm 2.85$ and, 29,527 African Americans, with a mean age of 69.1 \pm 2.83 from 2006-2008. During 20092011, the sample included men and women; 187,127 Caucasians, with a mean age of $69.26 \pm 2.87$ and, 31,063 African Americans, with a mean age of $69.13 \pm 2.86$. Inpatient and outpatient files from the Medicare database were used to collect data on the number of hospitalizations, the length of stay, the number of lower limb amputations, in-hospital mortality, the number of discharges to skilled nursing facilities, the number of doctors' visits, and the number of physical therapy visits for both groups. Other factors were also evaluated, such as the presence of hypertension, kidney disease, and depression.

\section{Procedures}

A systematic review of the literature was performed with focus on disparities in healthcare regarding African Americans compared to their Caucasian counterparts. Disparities were broken down into classifications of disorders concerning cardiac diseases, mental illness, endocrine disorders, musculoskeletal disorders, metabolic disorders, and healthcare provider interactions. A detailed literature review also was utilized to help identify the specific socioeconomic factors that have potentially led to increasing disparities in the occurrence of diabetes and its related complications in the African American population compared to Caucasians. Factors such as education level, amount of income, rates and incidence of incarceration, and mortality were examined in order to validate any current inequalities between the two groups.

\section{Results}

Table 1 shows a comparative analysis of secondary complications of Type 2 diabetes mellitus that arise in Caucasian and African American populations diagnosed with Type 2 diabetes mellitus from 2006 to 2008. Table 2 shows the same data from 2009 to 2011. African Americans displayed more complications than Caucasians in almost all categories of this study, with the exception of depression. A comparative study analyzing the same parameters was conducted for 2009 to 2011, and indicated that African Americans were still more likely to suffer from Type 2 diabetes mellitus related complications than Caucasians. However, the 2009 to 2011 study demonstrated a reduction in the number of African Americans and Caucasians diagnosed with hypertension, as well as a decreased in hospital mortality rate when

Table 1. Secondary Complications in Diabetic Patients among Caucasians and African Americans from 2006-2008.

\begin{tabular}{|l|l|l|l|}
\hline & Caucasians & $\begin{array}{l}\text { African- } \\
\text { Americans }\end{array}$ & P-Value \\
\hline Number of Hospital Stays (mean, sd) & $2.63 \pm 2.49$ & $3.05 \pm 2.97$ & $<.0001$ \\
\hline Length of Hospital Stay (mean, sd) & $15.61 \pm 22.99$ & $21.11 \pm 29.11$ & $<.0001$ \\
\hline Lower Limb Amputation & $0.7 \%$ & $1.6 \%$ & $<.0001$ \\
\hline Discharged to Skilled Nursing Facility & $9.7 \%$ & $11.9 \%$ & $<.0001$ \\
\hline In Hospital Mortality & $3.2 \%$ & $4.4 \%$ & $<.0001$ \\
\hline Hypertension & $79.3 \%$ & $86.4 \%$ & $<.0001$ \\
\hline Depression & $7.0 \%$ & $5.2 \%$ & $<.0001$ \\
\hline Kidney Disease & $17.6 \%$ & $26.6 \%$ & $<.0001$ \\
\hline Stroke & $26.3 \%$ & $26.9 \%$ & $<.0001$ \\
\hline Number of Physician Visits (mean, sd) & $24.72 \pm 20.37$ & $21.28 \pm 18.64$ & $<.0001$ \\
\hline Number of Physical Therapy (mean, sd) & $25.15 \pm 41.42$ & $19.56 \pm 34.73$ & $<.0001$ \\
\hline
\end{tabular}

Table 2. Secondary Complications in Diabetic Patients among Caucasians and African Americans from 2009-2011.

\begin{tabular}{|l|l|l|l|}
\hline & Caucasians & African-Americans & P-Value \\
\hline Number of Hospital Stays (mean, sd) & $2.60 \pm 2.51$ & $3.05 \pm 3.03$ & $<.0001$ \\
\hline Length of Hospital Stay (mean, sd) & $15.14 \pm 22.43$ & $20.58 \pm 29.28$ & $<. .0001$ \\
\hline Lower Limb Amputation & $0.7 \%$ & $1.5 \%$ & $<.0001$ \\
\hline Discharged to Skilled Nursing Facility & $9.9 \%$ & $12.1 \%$ & $<.0001$ \\
\hline In Hospital Mortality & $2.9 \%$ & $3.9 \%$ & $<.0001$ \\
\hline Hypertension & $78.7 \%$ & $85.3 \%$ & $<.0001$ \\
\hline Depression & $8.2 \%$ & $6.0 \%$ & $<.0001$ \\
\hline Kidney Disease & $21.0 \%$ & $29.8 \%$ & $<.0001$ \\
\hline Stroke & $25.2 \%$ & $26.6 \%$ & $<. .0001$ \\
\hline Number of Physician Visits (mean, sd) & $25.20 \pm 20.93$ & $21.93 \pm 19.33$ & $<. .0001$ \\
\hline Number of Physical Therapy (mean, sd) & $29.38 \pm 48.98$ & $22.47 \pm 43.65$ & $<. .0001$ \\
\hline
\end{tabular}

compared to data from 2006-2008 study. Interestingly, the rates of depression, kidney disease, and stroke increased slightly in both African Americans and Caucasians when comparing 2009-2011 data with the data collected from 2006-2008. Another important finding is that when comparing the 2006-2008 data to the 2009-2011 data is that the number of physical therapy visits increased in both populations, while the number of physician visits stayed relatively the same.

\section{Discussion and conclusions}

The rapidly increasing prevalence of type 2 diabetes is a growing concern for both Caucasians and African Americans. However, research shows that African Americans are more susceptible to type 2 diabetes mellitus than Caucasians. In the United States, members of minorities are nearly twice as likely to have diabetes as compared to Caucasians [4]. African Americans have a stronger genetic susceptibility to diabetes because of their increased risk for diabetic risk factors, such as being weight gain, high blood pressure, and sedentary lifestyles [4]. The research indicates that African Americans are more disinclined to follow up with their physicians to control their diabetic state, and consequently are at increased risk to suffer from secondary complications of the disease, such as amputations, hypertension, and kidney disease. These comorbidities result in African Americans experiencing increased length of hospital stays, more frequent hospital stays, and increased in-hospital mortality. African Americans are more likely to suffer from obesity, sedentary lifestyle, and low socioeconomic status than Caucasians. These are all risk factors that could lead to the development and potential progression of diabetes mellitus.

In order to understand the physical and mental effects of healthcare disparities there further needs to be an understanding of why such disparities exist. One of the first issues that should be examined is the social economic reasons such disparities exist. According to Williams and Mohammed, as compared to Caucasians, African Americans who have graduated from college are more likely to experience job loss; those who are employed have been shown to experience occupational hazards and carcinogens after subsequent job training and education. African Americans also have decreased capital at every level of income and decreased purchasing power due to the costs of an extensive range of goods and services that are higher in African Americans communities [5]. Secondly we must look at the cultural beliefs as it concerns aspects that influence food choices, dietary intake, and nutrition related attitudes among African American. In a study by James, six groups consisting of both males and females were interviewed on their thoughts of "healthiness", body image, and culture. The conclusions made by the groups were that becoming healthy meant giving up foods that taste good and that 
were more expensive. It was also discovered that the lack of a support system and knowledge had an influence in creating a healthy lifestyle [6]. The conclusions were made that the information gathered in the study should be the basis of how to formulate programs and ideas of living healthy within the African American community.

Finally we must look at some of the social factors that lead to a decline in African Americans male representation within the studies. According to the Centers for Disease Control the mortality rates for African American males are at a greater disproportion than there Caucasian counterparts as well as African Americans females. Both diabetes and heart disease are among the top 10 reasons for mortality rates within the African Americans male population. Other things to consider are the rates of homicide and incarceration among this population. Homicide rates in 2010 among non-Hispanic, African American males $10-24$ years of age $(51.5$ per 100,000$)$ surpassed those of Hispanic males (13.5 per 100,000) and non-Hispanic, White males in the same age group $(2.9$ per 100,000$)$ [7]. Incarceration rate within the African Americans community also affect the sample size of the populations studied. African Americans make up nearly 1 million of the total 2.3 million incarcerated population [8]. African Americans are incarcerated at nearly six times the rate of Caucasian, in 2009 Black non-Hispanic males were incarcerated at a rate of 4,749 inmates per 100,000, 6 times higher than white non-Hispanic males [9].

There continued to be mixed results in the literature when trying to explain why disparities exist between African Americans and Caucasians. Another explanation for the disparities was related to the quality of care patients receive from healthcare providers depending on their race. The American Diabetes Association established guidelines for diabetes process measures that include $\mathrm{HbAlc}$ testing twice a year, annual dilated eye examinations, and annual foot examinations. When analyzing the data of patients receiving care for diabetes, the percentage of African Americans receiving all three of the recommended diabetes care measures declined from $42.8 \%$ in 2002 to $31.7 \%$ in 2007 [1].

Also, the Agency for Healthcare Research and Quality published a report in 2006 stating that minority groups, including AfricanAmericans, with diabetes received poorer quality of care compared with Caucasians [10]. Their research included 22 measures of quality of care to assess the treatment received by the patients. In contrast to their findings, a recent study used data from 1997 to 2003 from Medicare beneficiaries demonstrated that improvements have been achieved in racial and ethnic disparities in the quality of care for patients with diabetes. The gap between Caucasians and African Americans narrowed in regards to the quality of care they received, which resulted in better outcomes due to the better care [11].

Another important factor that could contribute to diabetes disparity between African Americans and Caucasians is the income status of the two populations. In the 2012 National Healthcare Diabetes Report, it was shown that individuals who were considered to be poor, low-income, and middle-income adults were less likely to receive the recommended care needed to control diabetes when compared to those considered to be high income adults [12]. Another statistic shown in the NHRQ indicated that individuals aged 40-59 were less likely to receive the advised care that they needed to control diabetes as compared to those aged 60 and over [12].

LaVeist et al. also conducted a similar study analyzing the income and education levels between african americans and caucasians suffering from diabetes. In their study they found that African Americans were more likely to have decreased income and a lower education level than caucasians [13]. This was thoguht to correlate with the number of undiagnosed cases or poorly controlled diabetes cases found in the African American population. Also, education status of the participants in this study was lower in the African American population, with $21.7 \%$ of the African American participants having less than a high school diploma, while only $15 \%$ of the caucasian population had less than a high school diploma [13]. Participants of this study lived in the same low income urban community and it was documented that both have access to the same healthcare [13].

Despite the increase in prevalence of diabetes and its related complications within the African American population, there was a significant decrease in the sample size of African American men. One study suggests that while life expectancy of Caucasian males increases after the age of 50, there is a 12-year difference when compared to African American males. Another potential explanation for the decrease in sample size for African American males is due to their higher incarceration rates compared to other races. Research shows that African American males are incarcerated nearly six times the rate of Caucasians.

Our findings show that although African Americans are more susceptible to diabetes, there are common characteristics that exist within Caucasian and African American communities including poor lifestyle choices, poor dietary habits, sedentary lifestyles, and inadequate education about the disease that can lead to an increased risk for developing diabetes. Further areas of interest for this study would be to eliminate the income barrier within studies to reflect the populations that are either low income or high income to determine if income is responsible for the decreased maintenance for diabetes and to determine if it is one of the major factors for the disparity between African Americans and Caucasians [14].

\section{References}

1. Wilkes A, Bordenave K, Vinci L, Peek M (2011) Addressing diabetes racial and ethnic disparities: lesson learned from quality improvement collaboratives. Diabetes Manag 1: 653-660. [Crossref]

2. Zhang JX, Huang ES, Drum ML, Kirchhoff AC, Schlichting JA, et al. (2009) Insurance status and quality of diabetes care in community health centers. Am J Public Health 99: 742-747. [Crossref]

3. Black S (2002) Diabetes, diversity, and disparity: what do we do with the evidence? Am J Public Health 92: 543-548. [Crossref]

4. Hayes AJ, Leal J, Kelman CW, Clarke PM (2010) Risk equations to predict life expectancy of people with Type 2 diabetes mellitus following major complications: a study from Western Australia. Diabet Med 28: 428-435. [Crossref]

5. Maty SC, James SA, Kaplan GA (2010) Life-course socioeconomic position and incidence of diabetes mellitus among blacks and whites: the Alameda county study, 1965-1999. Am J Public Health 100: 137-145. [Crossref]

6. Williams DR, Mohammed SA (2009) Discrimination and racial disparities in health evidence and needed research. J Behav Med 32: 20-47. [Crossref]

7. James DC (2004) Factors influencing food choices, dietary intake, and nutrition related attitudes among African American: Application of a culturally sensitive model. Ethn Health 9: 349-367. [Crossref]

8. Centers for Disease Control and Prevention. Black or African American Populations. 10 Feb. 2014. Web. 22 Sept. 2014.

9. “Criminal Justice Fact Sheet.” NAACP. N.p., n.d. Web. 22 Sept. 2014

10. West HC (2010) Prison Inmates at Midyear 2009 - Statistical Tables (n.d.): n. pag. U.S Department of Justice. June 2010. Web.

11. Richard P, Alexandre PK, Lara A, Akamigbo AB (2011) Racial and ethnic disparities in the quality of diabetes care in a nationally representative sample. Prev Chronic Dis 8: A142. [Crossref]

12. Trivedi AN, Zaslavsky AM, Schneider EC, Ayanian JZ (2005) Trends in the quality 
of care and racial disparities in Medicare managed care. $N$ Engl J Med 353: 692-700. [Crossref]

13. U.S. Department of Health and Human Services (2013) 2012 National healthcare and disparities report. Agency for Healthcare Research and Quality.
14. LaVeist TA, Thorpe RJ Jr, Galarraga JE, Bower KM, Gary-Webb TL (2009) Environmental and socio-economic factors as contributors to racial disparities in diabetes prevalence. J Gen Intern Med 24: 1144-1148. [Crossref]

Copyright: (C2016 Shetty A. This is an open-access article distributed under the terms of the Creative Commons Attribution License, which permits unrestricted use, distribution, and reproduction in any medium, provided the original author and source are credited. 